Jorge Dueñas Ramírez

\title{
Mecanismos de Ruptura Bloco - Flexural em Maciços \\ Rochosos: Caso do Talude Norte da Mina de Tintaya (Peru)
}

Dissertação de Mestrado

Dissertação apresentada ao departamento de Engenharia Civil da PUC-Rio como parte dos requisitos para obtenção do título de mestre em Engenharia Civil: Geotecnia

Orientador: Eurípides Vargas Jr

Co - orientador: Rodrigo Figueiredo

Rio de Janeiro, agosto de 2006. 
Jorge Dueñas Ramírez

\section{Mecanismos de Ruptura Bloco - Flexural em Maciços Rochosos: Caso do Talude Norte da Mina de Tintaya (Peru)}

Dissertação de Mestrado

Dissertação apresentada ao Programa de Pósgraduação em Geotecnia da PUC-Rio como requisito parcial para obtenção do grau de Mestre em Engenharia Civil. Aprovada pela Comissão Examinadora abaixo assinada:

Prof. Eurípedes do Amaral Vargas Jr Orientador e Presidente Departamento de Engenharia Civil - PUC-Rio

Prof. Rodrigo Peluci de Figueiredo Co - orientador Universidade Federal de Ouro Preto - UFOP

Prof. Emílio Velloso Barroso Universidade Federal do Rio de Janeiro - IG/UFRJ

João Luiz Elias Campos Tecgraf - PUC-Rio José Eugênio Leal Coordenador Setorial do Centro Técnico Científico - PUC-Rio

Rio de Janeiro, 10 de agosto de 2006. 
Todos os direitos reservados. É proibida a reprodução total ou parcial do trabalho sem autorização da universidade, do autor e do orientador.

\section{Jorge Dueñas Ramírez}

Graduou-se em Geologia de Engenharia em 1999 pela Universidad Nacional de San Agustín (UNSA-Peru). Trabalhou na área de Hidrocarbonetos e Geotecnia tais como: PERUPETRO S.A., Sociedad Minera Cerro Verde SAA., Volcan Cia minera SAA, BHPBilliton Tintaya S.A., e Xstrata Copper Tintaya S.A., Ingressou em 2004 no curso de mestrado em Engenharia Civil da Pontifícia Universidade Católica do Rio de Janeiro, na área de Geotecnia, desenvolvendo dissertação de mestrado na linha de pesquisa Mecânica das Rochas.

Ficha Catalográfica

Ramírez, Jorge Dueñas

Mecanismo de Ruptura por Tombamento Bloco Flexural; Caso Talude Nor- Oeste - Mina Tintaya (Peru) / Jorge Dueñas Ramírez; Orientadores: Eurípedes do Amaral Vargas Jr. e Rodrigo Peluci de Figueiredo - Rio de Janeiro: PUC, Departamento de Engenharia Civil, 2006.

v., 150 f.: il. ; $29,7 \mathrm{~cm}$

Dissertação (mestrado) - Pontifícia Universidade Católica do Rio de Janeiro, Departamento de Engenharia Civil.

Inclui referências bibliográficas.

1. Engenharia civil - Teses. 2. Tombamento blocoflexural. 3. Maciço rochoso. 4. Descontinuidade. 5. Teoria de Cosserat. I. Vargas Jr, Eurípedes do Amaral. II. Figueiredo, Rodrigo Peluci de. III. Pontifícia Universidade Católica do Rio de Janeiro. Departamento de Engenharia Civil. IV. Título. 
A Deus, por ter me dado saúde.

A meus queridos pais: Juan de Dios e Encarnación, por terem me dado educação.

A María Amparo e Lucía Alejandra, por me darem felicidade.

A meus Irmaõs pelo apoio durante meus estudos do mestrado. 


\section{Agradecimentos}

A Deus e a Nossa Senhora pelo dom da vida e cuja fé neles, me deu a força necessária para vencer com lucidez todos obstáculos que tive.

Aos Professores Eurípides Vargas Jr., Rodrigo Figueiredo e João Luiz, pela orientação e guia durante esta pesquisa.

Aos meus queridos pais, Juan de Dios e Encarnación, pelo grande incentivo, mas torcendo muito pelo meu sucesso na minha Universidade.

A María Amparo, Lucía Alejandra e Valeria Gabriela que são a primeira e única razão da minha existência.

A meus Irmaõs: Nieves, Freddy, Jesús, Chela, Arístides e Amelia pelo apoio durante meus estudos do mestrado.

Aos meus professores, Eurípides Vargas Jr, Celso Romanel, Alberto Sayão, Sergio Fontoura, Franklin Antunes, Paulo Batista, Djenane Pamplona e Luciano Medeiros, pelos conhecimentos transmitidos, pela paciência que tiveram comigo e principalmente pelos inúmeros conselhos que me serão úteis por toda a vida.

Aos amigos, Ana Carolina, Ana Lúcia, Ana Vanessa, Álvaro, Christiano, Elisângela, Jackeline, Luis Alonso, Maria Bernardete, Roberth Apolinar, Taíse, Tania e Ygor, pelos bons momentos juntos durante o mestrado, aos amigos: Nelly Rubio e Álvaro Vianna pela revisão do presente tese. Alêm disso, gostaria agradecer a um gran amigo, Jhoan Paredes pelo apoio no Rio, durante o desenvolvimento da tese.

A secretária de pós-graduação Ana Roxo e Rita de Cássia pela grande ajuda e amizade nestes anos de mestrado;

Aos funcionários do departamento de Engenharia Civil, Fátima, Lenilson, Cristiano, pela dedicação e amizade que sempre tiveram comigo;

A BHP Billiton Tintaya S.A. e Xstrata Copper Tintaya S.A. pelo apoio e autorização em fazer o presente trabalho de investigação. Aos funcionários de Servicio Técnicos pelo apoio técnico e boa convivência na área Edgardo Orderique e Jorge Vargas.

Ao profesor Pablo Meza da Universidad Nacional deSan Agustín (UNSA - Perú) pelo apoio e incentivo em estudar Geotecnia.

Meus sinceros agradecimentos a todas aquelas pessoas não citadas, mas que de alguma forma contribuíram para o sucesso deste trabalho.

Ao povo do Brasil por ter me dado a oportunidade de estudar o mestrado. 


\section{Resumo}

Ramírez, Jorge Dueñas; Vargas Jr., Eurípedes do Amaral; Figueiredo, Rodrigo Peluci de. Mecanismo de Ruptura por Tombamento Bloco Flexural; Caso Talude Nor-Oeste - Mina Tintaya (Peru). Rio de Janeiro, 2006. 150 p. Dissertação de Mestrado - Departamento de Engenharia Civil, Pontifícia Universidade Católica do Rio de Janeiro.

Esta tese apresenta o fenômeno de ruptura por tombamento bloco-flexural, que acontece na zona NW da mina Tintaya. O objetivo do presente estudo é contribuir o conhecimento de este tipo de fenômeno de ruptura, quantificando as características do maciço rochoso com as técnicas da mecânica das rochas. $\mathrm{O}$ trabalho inicia-se por uma caracterização geral da zona de estudo, com especial relevo das propriedades geomecánicas das descontinuidades presentes, pelo que foram desenvolvidos mapeamentos das caras de bancada e a amostragem da rocha intacta, esta data permitiu estabelecer correlações dos parâmetros geomecânicos e fazer a análise cinemática da zona de estudo. Após de fazer a caracterização e avaliação das características das descontinuidades, foi definido o tombamento bloco-flexural, pelo jeito das descontinuidades presentes no maciço, já que este tipo de ruptura é mais complexo do que os outros tipos de tombamento, porque é uma combinação de tombamento e deslizamento dos blocos. Em vez da ruptura flexural de colunas contínuas, neste caso o tombamento é resultado de deslocamentos acumulados das juntas transversais. Com o objetivo de fazer uma análise numérica, obtou-se por um modelo contínuo-equivalente que inclui os efeitos de orientação e espaçamento das juntas é o modelo de plasticidade de Cosserat. O contínuo de Cosserat, conhecido também como contínuo micropolar acrescenta os graus de liberdade de rotação ao contínuo convencional. Pelo que foi analisado este tipo de ruptura através de uma modelagem computacional com um programa de elementos finitos feito na PUC-Rio, assim, tornar possível a modelagem computacional com a teoria do continuo de Cosserat.

\section{Palavras-chave}

Tombamento bloco-flexural, maciço rochoso, descontinuidade, Teoria de Cosserat 


\section{Abstract}

Ramírez, Jorge Dueñas; Vargas Jr., Eurípedes do Amaral; Figueiredo, Rodrigo Peluci de (Advisors). Block-Flexure Toppling Mechanism; Case NW Slope Tintaya's Mine. Rio de Janeiro, 2006. $150 \mathrm{p}$. MSc. Dissertation - Department of Civil Engineering, Pontifícia Universidade Católica do Rio de Janeiro.

This thesis presents the block - flexural toppling failure phenomenon, which happens at NW zone of the Tintaya's mine. The objective of the present study is to contribute the knowledge of this type of phenomenon of failure, quantifying the characteristics of rock mass with the techniques of the rock mechanics. The work begins for a general characterization of the zone, with special relief of the geomechanical properties of the discontinuities, which were developed the window sampling (bench face mapping) and sampling of the intact rock, this information allowed to establish correlations of the geomechanical parameters and make feasible kinematics analysis from the zone of study. After doing the rock mass characterization and evaluation of the characteristics of the discontinuities, the block - flexural toppling failure was defined, on those long column rocks crossed by numerous huge subhorizontal joints. The block-flexural type failure is a complex phenomenon compared with other types of toppling failure, because it is a combination of pure toppling and sliding. With the intention of numerical analysis, I have been proposed analyzing this rupture for a continuum - equivalent model of Cosserat, that includes the orientation and the discontinuity's spacing. This model is known also as continuum micropolar, which adds the degrees of freedom of rotation to a conventional continuum. It was analyzed this type of failure through a computational modeling with a program of finite elements made in the PUC-Rio, so, the computational modeling possible with the theory of continuum of Cosserat.

\section{Keywords}

Block-flexure toppling, Rock mass, Discontinuity, Cosserat Theory 


\section{Sumário}

1. Introdução 15

$1.1 \quad$ Objetivos e Estratégia do trabalho de pesquisa 16

1.2 Relevância do Trabalho de Pesquisa 17

1.3 Organização de Dissertação 18

2. Propriedades Geomecânicas do Maciço Rochoso 19

$\begin{array}{lll}2.1 & 19\end{array}$

2.2 Efeito Escala e Resistência 20

$2.3 \quad$ Resistência das Descontinuidades 23

$2.4 \quad$ Rigidez das Descontinuidades 27

$2.5 \quad$ Critério de ruptura generalizada de Hoek-Brown 30

3. Análise dos Mecanismos de Ruptura de tombamento 36

3.1 Generalidades 36

3.2 Tombamento Flexural 38

3.3 Tombamento de Blocos 38

3.4 Tombamento bloco-flexural 39

$3.5 \quad$ Revisão de Literatura 40

3.6 Métodos de Análise 42

3.6.1 Modelos Físicos 42

3.6.2 Análise Cinemático 46

3.6.3 Análise de equilíbrio limite 45

4. Modelagem Numérica $\quad 54$

$4.1 \quad$ Introdução 54

4.2 A Mecânica Generalizada de Cosserat e o Modelo Elastoplástico para Meios Estratificados $\quad 56$

4.2.1 A Mecânica Generalizaa de Cosserat 56

4.2.1.1 Cinemática $\quad 57$

4.2.1.2 Estática 58

4.2.1.3 Equações Constitutivas 58

4.2.1.3.1 Elasticidade Linear Isotrópica 58

4.2.1.3.2 Elastoplasticidade 60

4.2.1.3.2.1 Modelo Elastoplático para Meios Estratificados 60

5. Instabilidade por Tombamento na mina Tintaya 64

$5.1 \quad$ Generalidades 64

$5.2 \quad$ Geologia da Mina Tintaya 66 
$\begin{array}{lll}5.3 & \text { Instabilidade zona NW de Tintaya } & 67\end{array}$

$\begin{array}{lll}5.4 & \text { Estrutura do Maciço Rochoso } & 70\end{array}$

$5.5 \quad$ Análise de Equilibrio Limite 72

5.6 Propriedades do Maciço Rochoso 76

$\begin{array}{lll}5.7 & \text { Modelagem Numérica } & 79\end{array}$

5.7.1 Resultados Obtidos 82

6. Conclusões e sugestões 85

6.1 Conclusões 85

6.2 Sugestões 86

$\begin{array}{ll}\text { Bibliografia } & 87\end{array}$

$\begin{array}{ll}\text { Anexo } 1 & 92\end{array}$

$\begin{array}{ll}\text { Anexo } 2 & 95\end{array}$ 


\section{Lista de Figuras}

Figura 1.1

Figura 1.2

Figura 2.1

Figura 2.2

Figura 2.3

Figura 2.4

Figura 2.5

Figura 2.6

Figura 2.7

Figura 3.1

Figura 3.2

Figura 3.3

Figura 3.4

Figura 3.5

Figura 3.6

Figura 3.7

Figura 3.8

Figura 4.1

Figura 4.2

Figura 4.3

Figura 4.4

Figura 5.1

Figura 5.2

Figura 5.3

Figura 5.4

Figura 5.5

Figura 5.6

Figura 5.7
Mapa de localização do distrito mineiro de Tintaya.

Mapa de localização dos puntos de monitoramento da zona 2 (NW) tajo Tintaya.

Diagrama idealizado mostrando transição desde rocha intacta até o maciço rochoso fraturado com o incremento do tamanho de amostra.

Relação entre à Geologia e as classes de resistência da rocha Relação entre as tensões de cisalhamento e normal sob uma superfície de ruptura para cinco diferentes condições geológicas.

Envoltoria de ruptura bilinieal para superficies múltiples.

Variação de valores medidos de ks com a escala e o nível das tensões normais.

Razão Kn / Ks em função em função de $\sigma_{n}$.

Relações entre as tensões principias máxima e mínima para os critérios de Hoek-Brown e equivalente de Mohr-Coulomb.

Tipos de ruptura por tombamento.

Mecanismo de ruptura e tombamento secundário.

Mecanismo de ruptura por tombamento flexural.

Modelo esquemático da base friction mostrando a barreira, o modelo simulado e a correia de lixa.

Análise cinemática para a ruptura pelo tombamento.

Condições de estabilidade em função da geometria dum bloco paralelepipédico sob acção do peso próprio.

Princípios do equilíbrio limite para a análise de tombamento.

Modelo para análise de equilíbrio-limite do tombamento flexural.

Ponto material em um contínuo de Cosserat 2D (a) e representação de $\gamma_{12}$ (b).

Equilíbrio de forças (a) e momentos (b) em um elemento infinitesimal num meio de Cosserat.

Parcelas simétrica e anti-simétrica do tensor de tensões-força de Cosserat (a) e representação gráfica de Mohr (b).

Geometria de um meio estratificado e tensões de Cosserat atuantes.

Zonificação Geomecânica da Mina Tintaya (Departamento Geotecnia, 2005).

Litotipos predominantes na Mina Tintaya (Departamento Geologia, 2005).

Mapa geológico da mina Tintaya.

Desenvolvimento de trincas na zona NW do talude mina Tintaya; (a) trinca desenvolvida na cota 3925, pode-se observar a direção do movimento das juntas desenvolvidas, (b) sistema de trincas desenvolvidas em material de rejeito e solo natural, na cota 4105.

Deslocamentos obtidos pelo programa de monitoramento, valor máximo dos deslocamentos verticais $4.5 \mathrm{~m}$ e valor médio dos deslocamentos verticais relativos: $2.5 \mathrm{~m}$

Análise cinemática da zona 2 , referentes às descontinuidades presentes no maciço rochoso.

Análise cinemática da zona 2 , referentes às descontinuidades da zona. 
Figura 5.8 Tombamento bloco-flexural definido na zona 2 nível 4030

Figura 5.9 Tombamento bloco-flexural no nível 3955, pode-se observar os deslocamentos das juntas transversais.

Figura 5.10

Figura 5.11 Tombamento bloco-flexural no nível 3910.

Comportamento pós-pico típico em função do GSI do maciço rochoso (Hoek \& Brown, 1997): (a) GSI >= 75, elasto-frágil perfeito; (b) $75<\mathrm{GSI}<=30$, elasto-plástico com amolecimento e (c) GSI < 30, elasto-plástico perfeito.

Figura 5.12 Modelo adotado da Mina Tintaya.

Figura 5.13

Malha de elementos finitos para o modelo da Mina Tintaya.

Figura 5.14

Campo de deslocamentos mostrando o possível mecanismo de ruptura por tombamento do talude.

Figura 5.15 Características típicas do padrão de deslocamentos em análises de tombamento flexural. 


\section{Lista de Símbolos}

\begin{tabular}{|c|c|}
\hline$\mu$ & Coeficiente de fricção \\
\hline$\sigma_{n}$ & Tensão normal aplicado \\
\hline$\tau$ & Resistência ao cisalhamento das juntas \\
\hline$\phi_{b}$ & Ângulo de fricção básico \\
\hline$i$ & Ângulo de inclinação da rugosidade (Patton) \\
\hline$c_{j}$ & Coesão aparente da junta \\
\hline$\phi_{r}$ & Ângulo de fricção residual \\
\hline JRC & Coeficiente de rugosidade de junta \\
\hline JCS & Resistência à compressão da rocha na superfície de fratura \\
\hline$\sigma^{\prime}$ & Tensão normal efetiva \\
\hline$\phi_{p}$ & Ângulo de fricção pico \\
\hline $\mathrm{Kn}$ & Rigidez normal da junta \\
\hline Ks & Rigidez transversal da junta \\
\hline$\tau_{p}$ & Tensão de cisalhamento pico \\
\hline$d_{h p}$ & Deslocamento das juntas \\
\hline$L$ & Escala da junta (limitada pelo espaçamento de outras juntas transversais) \\
\hline$\sigma_{1}^{\prime}$ & Tensão principal efetiva máxima ou maior \\
\hline$\sigma_{2}^{\prime}$ & Tensão principal efetiva intermediária \\
\hline$\sigma_{3}^{\prime}$ & Tensão principal efetiva mínima ou menor \\
\hline$\sigma_{1}$ & Tensão principal máxima ou maior \\
\hline$\sigma_{2}$ & Tensão principal intermediária \\
\hline$\sigma_{3}$ & Tensão principal mínima ou menor \\
\hline$\sigma_{x}$ & Tensão aplicada na direção X \\
\hline$\sigma_{y}$ & Tensão aplicada na direção Y \\
\hline$\sigma_{x}$ & Tensão aplicada na direção Z \\
\hline$\sigma_{m}^{\prime}$ & Tensão efetiva média \\
\hline$E$ & Módulo de Young \\
\hline$m_{b}$ & $\begin{array}{l}\text { Valor reduzido da constante do material mi ou constante do maciço } \\
\text { rochoso }\end{array}$ \\
\hline s, a & Constantes para o maciço rochoso \\
\hline GSI & Índice de resistência geológica \\
\hline RMR & Rock Mass Rating \\
\hline $\mathrm{D}$ & Fator de perturbação \\
\hline
\end{tabular}




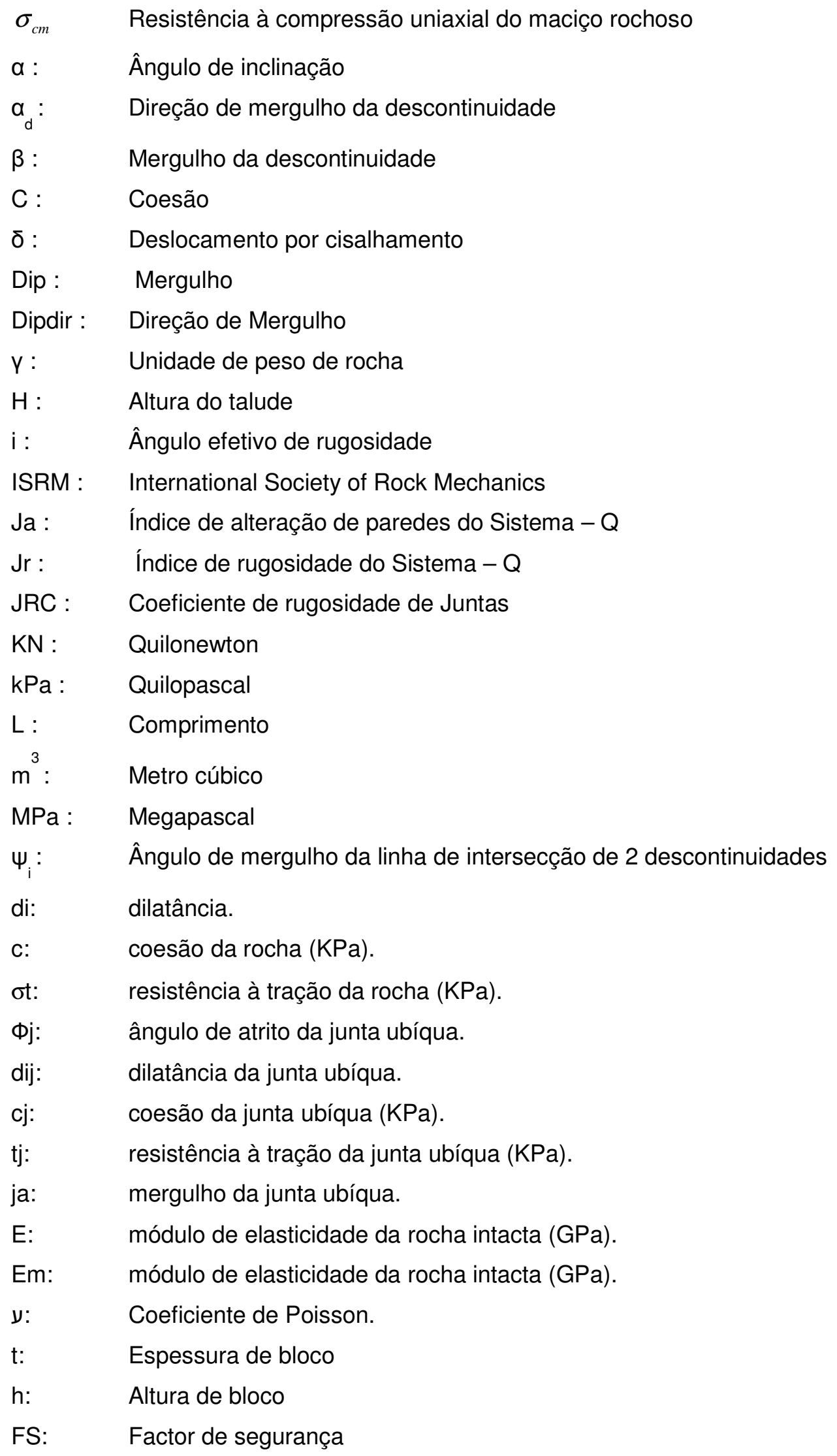


$(x 1, x 2), x i(i=1,2) \quad$ Coordenadas de um ponto material em um contínuo de Cosserat 2D.

rij e кi: Deformação em um contínuo de Cosserat.

$e_{i j}: \quad$ Tensor alternante a 2D

$\partial_{i}():$.$\quad Gradiente na i$-ésima direção espacial

Fi: $\quad$ Forças e o momento de massa

$\Theta$ : $\quad$ Momento de massa.

$\lambda$ e G: $\quad$ Parâmetros clássicos de Lamé.

Gc: $\quad$ Módulo de cisalhamento anti-simétrico.

B: $\quad$ Módulo de flexão.

$\hat{c}$ : Coesão-momento (força/comprimento) de Cosserat.

$\lambda s, \lambda t$ e $\lambda$ Multiplicadores plásticos relativos.

$f_{s} \quad$ Função de escoamento.

$g_{s} \quad$ Função potencial plástico. 